\title{
Postpartum exacerbation of antenatal COVID-19 pneumonia in 3 women
}

\author{
Peng An MD, Bradford J. Wood MD, Wei Li MD, Min Zhang MD, Yingjian Ye MD
}

— Cite as: CMAJ 2020 June 1;192:E603-6. doi: 10.1503/cmaj.200553; early-released May 6, 2020

\section{Case 1}

A31-year-old pregnant woman (38 $w k+2 d)$ presented to the obstetrics and gynecology team with a 5-day history of cough, low-grade fever (maximum temperature $37.8^{\circ} \mathrm{C}$ ) and intermittent shortness of breath. She also described bilateral lower-leg edema for 2 days. Because she reported contact with a potentially infected person from Wuhan, China, the epicentre of the coronavirus disease 2019 (COVID-19) outbreak, we placed her immediately in respiratory isolation.

The patient's oxygen saturations were 90\%-94\%, respiratory rate was 28 breaths/min, heart rate was 90 beats/min and blood pressure was $160 / 100 \mathrm{~mm} \mathrm{Hg}$ at the time of admission. Results of initial laboratory tests were as follows: leukocyte count 5.1 (reference range $3.5-9.5) \times 10^{9} / \mathrm{L}$, lymphocyte count 1.05 (reference range $1.1-3.2) \times 10^{9} / \mathrm{L}$ and $\mathrm{C}$-reactive protein (CRP) level 7.1 (reference range $0-8) \mathrm{mg} / \mathrm{L}$. Real-time polymerase chain reaction (PCR) testing for severe acute respiratory syndrome coronavirus 2 (SARS-CoV-2) infection was positive. Fetal ultrasonography and maternal echocardiography were normal.

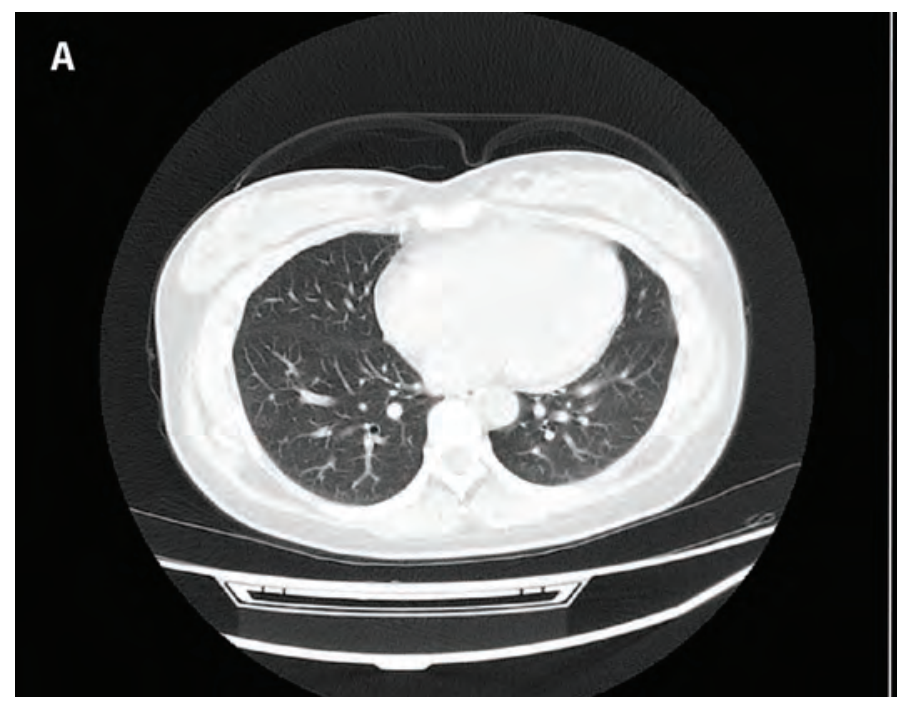

\section{KEY POINTS}

- Postpartum exacerbation of coronavirus disease 2019 symptoms may be sudden, within hours of delivery.

- Acute clinical deterioration of women with severe acute respiratory syndrome coronavirus 2 (SARS-CoV-2) infection who have recently given birth may be associated with changes in findings on chest computed tomography.

- Delayed hospital discharge or close community follow-up should be considered for women with SARS-CoV-2 infection who have recently given birth.

We performed low-dose computed tomography (CT) of the chest to exclude pulmonary embolism and pneumonia; CT results were normal (Figure 1A). After a failed induction of labour, the patient underwent a cesarean delivery, delivering a healthy boy weighing $4.2 \mathrm{~kg}$. The newborn tested negative for SARSCoV-2 by real-time PCR throat and anal swabs. After delivery, we prescribed the patient an antiviral treatment typically used for influenza, umifenovir.

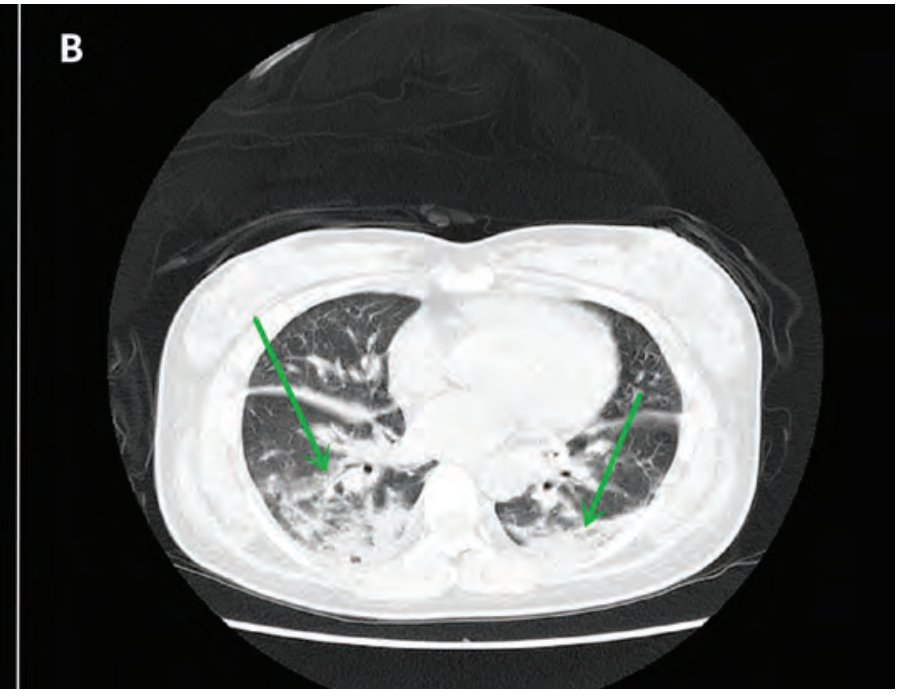

Figure 1: (A) Prepartum chest computed tomography (CT) scan in a 31-year-old pregnant woman. Results were normal, despite symptomatic features consistent with severe acute respiratory syndrome coronavirus 2 infection. Lead protective clothing was wrapped around the abdomen to reduce scatter radiation. (B) Postpartum chest CT scan, 28 hours after delivery, showing interval development of scattered bilateral ground-glass densities and peribronchial infiltrates in the posterior aspect of the upper and lower lobes (arrows), consistent with viral pneumonia. 

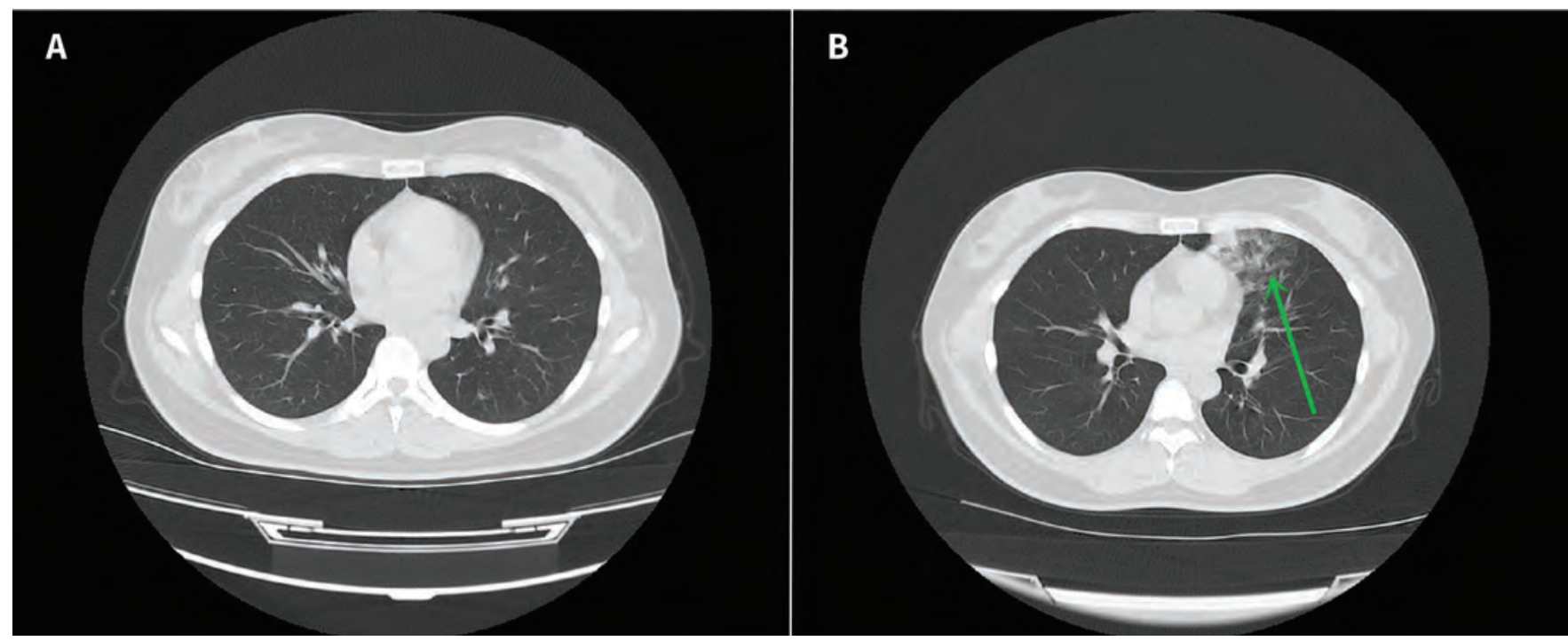

Figure 2: (A) Prepartum chest computed tomography (CT) scan in a 33-year-old pregnant woman. Results were normal, despite symptomatic features consistent with severe acute respiratory syndrome coronavirus 2 infection. Lead protective clothing was wrapped around the abdomen to reduce scatter radiation. (B) Postpartum CT scan, 58 hours after delivery, showing peripheral ground-glass infiltrates in the left lung (arrow), consistent with viral pneumonia.

Immediately after delivery, she had a mild cough. At 28 hours postpartum, however, sudden severe dyspnea developed, with oxygen saturation of $71 \%$ and a temperature of $39^{\circ} \mathrm{C}$. Echocardiography showed normal cardiac function. Repeat blood tests showed the following: leukocyte count 12.1 (reference range 3.59.5) $\times 10^{9} / \mathrm{L}$, neutrophil count 9.51 (reference range $1.8-6.3$ ) $\times 10^{9} / \mathrm{L}$, neutrophil percentage $90.0 \%$ (reference range $40 \%-75 \%$ ), lymphocyte count 0.75 (reference range $1.1-3.2$ ) $\times 10^{9} / \mathrm{L}$, lymphocyte percentage $7.2 \%$ (reference range $20 \%-50 \%$ ) and CRP level 15.3 (reference range $0-8) \mathrm{mg} / \mathrm{L}$. We repeated chest $\mathrm{CT}$, which showed the development of scattered bilateral ground-glass densities (Figure 1B). The patient subsequently received highflow oxygen to maintain oxygen saturations at $88 \%$ or greater, convalescent plasma transfusion and oral lopinavir-ritonavir 200/50 mg 2 tablets daily. After 4 days of treatment in hospital, she made a full recovery.

\section{Case 2}

A 33-year-old pregnant woman (39 $w k+4 d)$ presented to the obstetrics and gynecology team with a 4-day history of fever (maximum temperature $38.2^{\circ} \mathrm{C}$ ), cough and fatigue. She had a history of exposure to a health care worker from Wuhan, who later received the diagnosis of asymptomatic SARS-CoV-2 infection and whose real-time PCR test was positive.

The patient's oxygen saturation was $99 \%$, respiratory rate was 21 breaths/min, heart rate was 75 beats/min and blood pressure was $155 / 110 \mathrm{~mm} \mathrm{Hg}$ at the time of admission. Real-time PCR testing was positive for SARS-CoV-2 infection. Results of initial blood tests and chest CT (Figure 2A) were normal. She delivered a healthy $4.0 \mathrm{~kg}$ boy by cesarean delivery. The newborn tested negative for SARS-CoV-2 infection by real-time PCR throat and anal swabs. The patient reported feeling well until 58 hours postpartum, when she developed sudden coughing, dizziness, short- ness of breath and hypoxia with oxygen saturations of $85 \%$. At this time, results of her blood tests were as follows: lymphocyte count 1.01 (reference range $1.1-3.2$ ) $\times 10^{9} / \mathrm{L}$, lymphocyte percentage $16.6 \%$ (reference range $20 \%-50 \%$ ) and CRP level 13.13 (reference range $0-8) \mathrm{mg} / \mathrm{L}$. Repeat CT was performed, which showed peripheral ground-glass infiltrates in the left lung (Figure 2B). The patient recovered after an 8-day hospital admission.

\section{Case 3}

A 25-year-old pregnant woman (38 wk +5 d) presented to our obstetrics and gynecology department with a 3-day history of fever, cough, chest tightness, shortness of breath and diarrhea. She had a history of exposure to a neighbour who was positive for SARS-CoV-2 infection on real-time PCR testing. Her oxygen saturation was $98 \%$, temperature was $37.5^{\circ} \mathrm{C}$, respiratory rate was 18 breaths $/ \mathrm{min}$, heart rate was 66 beats/min and blood pressure was $148 / 95 \mathrm{~mm} \mathrm{Hg}$ at the time of presentation. Results of the initial blood tests were as follows: leukocyte count 4.5 (reference range 3.5-9.5) $\times 10^{9} / \mathrm{L}$, lymphocyte count 0.98 (reference range $1.1-3.2$ ) $\times 10^{9} / \mathrm{L}$, lymphocyte percentage $18.1 \%$ (reference range $20 \%-50 \%$ ) and CRP level 9.1 (reference range $0-8$ ) $\mathrm{mg} / \mathrm{L}$. She was positive for SARS-CoV-2 infection on real-time PCR testing. On admission, CT was performed and showed mild and subtle haziness of early ground-glass infiltrates in the right peripheral lung (Figure $3 \mathrm{~A}$ ).

Cesarean delivery was performed, and she delivered a healthy $4.4 \mathrm{~kg}$ boy, who tested negative for SARS-CoV-2 infection by realtime PCR throat and anal swabs. She reported feeling well until 81 hours postpartum, when she developed a progressive cough and her oxygen saturation suddenly dropped to $80 \%$. Repeat CT was performed, which showed marked progression of the peripheral infiltrates in the right lung with consolidation of the ground-glass infiltrates (Figure 3B). After 10 days of supportive care in hospital, the patient was discharged. 

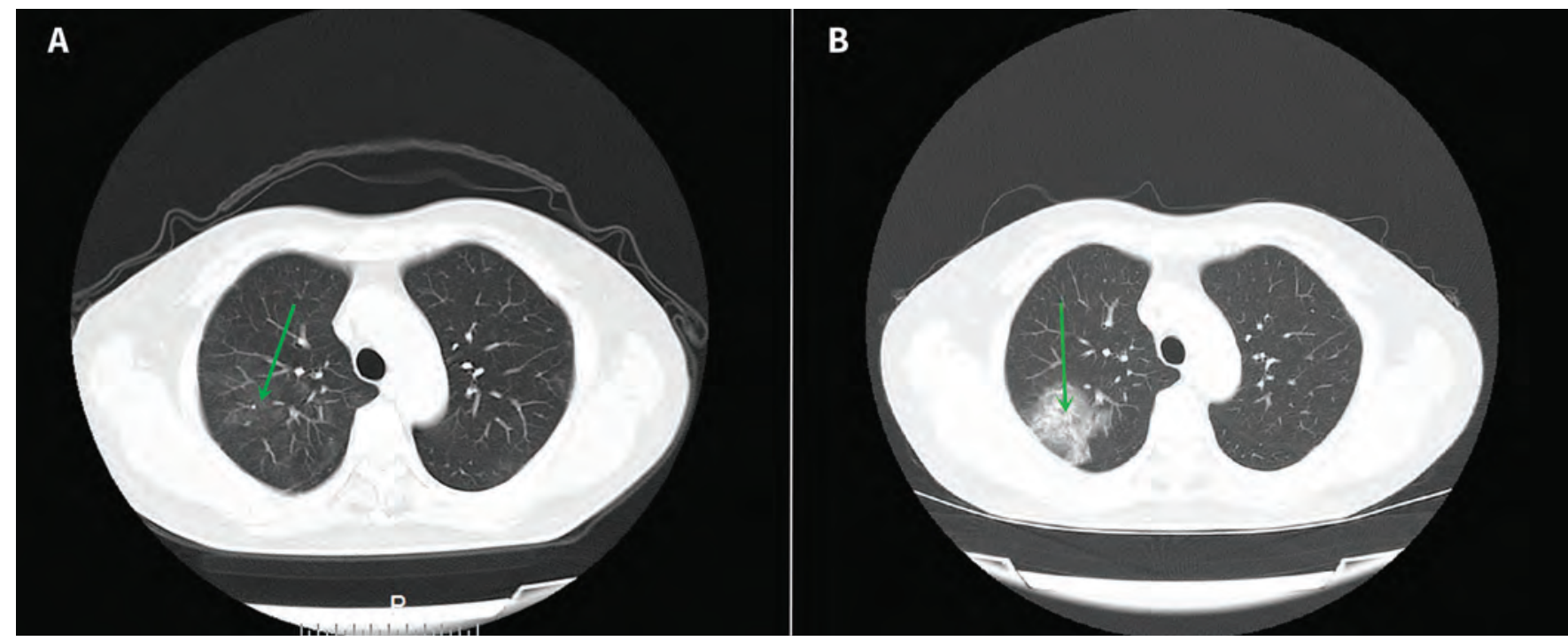

Figure 3: (A) Prepartum chest computed tomography (CT) scan in a 25-year-old pregnant woman showing mild and subtle haziness of early groundglass infiltrates in the right peripheral lung (arrow). Lead protective clothing was wrapped around the abdomen to reduce scatter radiation. (B) Postpartum CT scan, 81 hours after delivery, showing marked progression of the peripheral infiltrates in the right lung with consolidation of the groundglass infiltrates (arrow) with peripheral haziness and a higher-attenuation centre.

\section{Discussion}

COVID-19 has been reported in pregnancy but is not well described in the immediate postpartum period. From Feb. 1 to Apr. 15, 2020, we established a retrospective case cohort of all women who delivered in a predefined administrative region containing 12 hospitals, including the Xiangyang First People's Hospital in Hubei, China. In total, 166 pregnant women tested positive for SARS-CoV-2 infection prepartum, of whom 153 (92.2\%) showed mild features only or were asymptomatic. Postpartum dyspnea developed in $13(7.8 \%)$ of the women, and we present the 3 cases of pregnant women with SARS-CoV-2 infection who developed severe postpartum pneumonia with acute decompensation. In all cases, postpartum chest CT showed radiographic features consistent with COVID-19 that were not present or very mild in prepartum chest CT scans. This case series shows that an acute exacerbation of maternal COVID-19 may occur in the postpartum period.

Recently published case series discuss clinical features and outcomes of pregnant women with suspected COVID-19. ${ }^{1-4}$ In 1 series of 13 mothers with confirmed SARS-CoV-2 infection postpartum, 2 had a dry cough and 6 had ground-glass opacity infiltrates on chest $\mathrm{CT} .{ }^{1}$ In another retrospective single-centre study, 7 pregnant (third-trimester) women who tested positive for SARS-CoV-2 infection had infiltrates on CT consistent with infection but did not require intensive care postpartum. ${ }^{2}$ Of a total of 20 reported patients, none developed postpartum acute decompensation. ${ }^{1,2}$ Two recent reports from the same institution in New York described 29 women who had been asymptomatic but tested positive for SARS-CoV-2 infection on screening just before delivery. ${ }^{3,4}$ Three women (out of 29) developed fever postpartum; in 2 of these women, the fever was thought to be related to endomyometritis, and in 1 woman, the fever was thought to be related to COVID-19. ${ }^{3,4}$
In our case series, the women showed postpartum respiratory distress with deoxygenation and sudden clinical decompensation 28-81 hours postpartum, associated with lymphopenia, elevated CRP and changes in chest CT consistent with SARS-CoV-2 infection. Physiologically, it is unclear why these women developed serious symptoms after delivery. We hypothesize this may have been because of hemodynamic, immunologic and plasma volume changes that interfered with normal hormonal and diuresis changes postpartum, which predispose to changes in pulmonary vasculature and, potentially, decompensation..$^{5-7}$ These changes may be exacerbated, furthermore, in immunocompromised hosts who have systemic inflammatory changes, such as those with COVID-19.

Given the paucity of data about mother-to-child transmission, and after multidisciplinary discussion, it was decided that all 3 women should deliver by cesarean as it was unknown whether vaginal delivery by a mother with SARS-CoV-2 infection would increase the risk to health care professionals who were present during delivery of contracting SARS-CoV-2. The 3 newborns delivered did not test positive for SARS-CoV-2 on real-time PCR, consistent with a case series showing no vertical transmission of the virus from mother to neonate in 9 cases. ${ }^{8}$ However, there are 2 separate reports of neonates who tested positive for SARS-CoV-2 infection on real-time PCR 16 hours and 36 hours after birth, but how these infections were transmitted remains unclear. ${ }^{2,9}$

These 3 cases show that normal peripartum chest CT, in the presence of mild symptoms, does not preclude an abrupt postpartum decompensation. Awareness of the potential for this to occur may guide obstetric and midwifery teams to closely monitor women who tested positive for SARS-CoV-2 infection immediately after delivery, and for days after. ${ }^{10-12}$ Consideration might be given to delay discharge in women with SARS-CoV-2 infection who have recently given birth. Health care professionals, furthermore, should ensure that new mothers who are discharged from hospital 
are aware of how to seek medical attention if they experience symptoms of COVID-19 after giving birth. If hospital follow-up is not possible, follow-up in the community for both mother and baby, days after discharge, could be prioritized for patients with SARS-CoV-2 infection to ensure safety in the postnatal period.

\section{References}

1. Yang H, Sun G, Tang F, et al. Clinical features and outcomes of pregnant women suspected of coronavirus disease. J Infect 2019 Apr. 12 [Epub ahead of print]. pii: S0163-4453(20)30212-7. doi: 10.1016/j.jinf.2020.04.003.

2. Yu N, Li W, Kang Q, et al. Clinical features and obstetric and neonatal outcomes of pregnant patients with COVID-19 in Wuhan, China: a retrospective, singlecentre, descriptive study. Lancet Infect Dis 2020 Mar. 24 [Epub ahead of print]. pii: S1473-3099(20)30176-6. doi: 10.1016/S1473-3099(20)30176-6.

3. Breslin N, Baptiste C, Gyamfi-Bannerman C, et al. COVID-19 infection among asymptomatic and symptomatic pregnant women: two weeks of confirmed presentations to an affiliated pair of New York City hospitals. Am J Obstet Gynecol MFM 2020 Apr. 9 [Epub ahead of print]. 100118. doi: 10.1016/j.ajogmf.2020.100118.

4. Sutton D, Fuchs K, D'Alton M, et al. Universal screening for SARS-CoV-2 in women admitted for delivery. N Engl J Med 2020 Apr. 13 [Epub ahead of print]. doi: 10.1056/NEJMc2009316

5. Chen S, Huang B, Luo DJ, et al. Pregnant women with new coronavirus infection: a clinical characteristics and placental pathological analysis of three cases. [article in Chinese]. Zhonghua Bing Li Xue Za Zhi 2020 Mar. 1 [Epub ahead of print]. 49:E005. doi: 10.3760/cma.j.cn112151-20200225-00138.

6. National Health Commission of the People's Republic of China. Interim diagnosis and treatment of 2019 novel coronavirus pneumonia. 7th ed. 2020 Mar. 3. Available: www.nhc.gov.cn/yzygj/s7653p/202003/46c9294a7dfe4cef80dc7f591 2eb1989.shtml (accessed 2020 Mar. 4).

7. Dashraath P, Wong JLJ, Lim MXK, et al. Coronavirus disease 2019 (COVID-19) pandemic and pregnancy. Am J Obstet Gynecol 2020 Mar. 23 [Epub ahead of print]. pii: S0002-9378(20)30343-4. doi: 10.1016/j.ajog.2020.03.021.

8. Chen $\mathrm{H}$, Guo J, Wang $\mathrm{C}$, et al. Clinical characteristics and intrauterine vertical transmission potential of COVID-19 infection in nine pregnant women: a retrospective review of medical records [published erratum in Lancet 2020;395:1038]. Lancet 2020;395:809-15.

9. Alzamora MC, Paredes T, Caceres D, et al. Severe COVID-19 during pregnancy and possible vertical transmission. Am J Perinatol 2020 Apr. 18 [Epub ahead of print]. doi: 10.1055/s-0040-1710050.

10. Chen L, Li Q, Zheng D, et al. Clinical characteristics of pregnant women with COVID-19 in Wuhan, China. N Engl J Med 2020 Apr. 17 [Epub ahead of print]. doi: 10.1056/NEJMc2009226

11. Panahi L, Amiri M, Pouy S. Risks of novel coronavirus disease (COVID-19) in pregnancy: a narrative review. Arch Acad Emerg Med 2020;8:e34.

12. Sutton D, Fuchs K, D'Alton M, et al. Universal screening for SARS-CoV-2 in women admitted for delivery. N Engl J Med 2020 Apr. 13 [Epub ahead of print]. doi: 10.1056/NEJMc2009316.

\section{Competing interests: None declared.}

This article has been peer reviewed.

The authors have obtained patient consent.

Affiliations: Departments of Radiology (An), Infectious Diseases (An, Zhang, Ye) and Respiratory and Critical Care (An, Zhang, Ye), Xiangyang First People's Hospital, Hubei University of Medicine, Hubei, China; Center for Interventional Oncology, Radiology and Imaging Sciences (Wood), National Institutes of Health (NIH) Clinical Center and National Cancer Institute, NIH, Bethesda, Md.; Department of Obstetrics and Gynecology (Li), Xiangyang First People's Hospital, Hubei University of Medicine, Hubei, China
Contributors: Peng An, Yingjian Ye and Bradford Wood conceived and drafted the manuscript. All of the authors contributed to the literature review, revised the manuscript critically for important intellectual content, approved the final version to be published and agreed to act as guarantors of the work. Peng An and Bradford Wood contributed equally to this work.

Funding: This report was supported in part by the NIH Center for Interventional Oncology and the NIH Intramural Research Program through a collaboration between Bradford Wood and Peng An.

Acknowledgements: The authors gratefully acknowledge Sheng Xu and Baris Turkbey for assistance with translating references.

Correspondence to: Min Zhang, lixyyy2015@163.com; Yingjian Ye, yeyingjian88@126.com 